\title{
Article
}

\section{A Review of Ontological Models Applied for the Assistance of Persons with Disabilities}

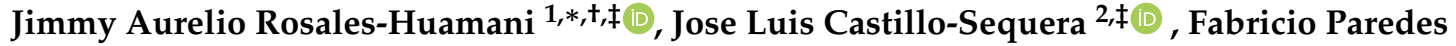 \\ Larroca ${ }^{1, \ddagger(\mathbb{D}, \text { Alberto Landauro Abanto }}{ }^{1, \ddagger}$ and Luis Zuloaga Rotta ${ }^{1, \ddagger}$ \\ 1 Multidisciplinary Sensing and Universal Accessibility Group, National University of Engineering, Lima \\ 15333,Peru; fabricio_paredes@hotmail.com, albertolandauro@yahoo.es, zuloaga_luis@uni.edu.pe \\ 2 Department of Computer Science, Higher Polytechnic School, University of Alcala, 28871 Alcalá de Henares, \\ Spain; jluis.castillo@uah.es \\ * Correspondence: jrosales@uni.edu.pe; Tel.: +51-1-381-5639 \\ $\ddagger \quad$ These authors contributed equally to this work.
}

\begin{abstract}
At least $15 \%$ of the population in the world has some type of disability. Unfortunately, this population has the problem of facing various accessibility barriers, to which technological barriers are also added. One of the most relevant obstacles is the one that arises as a result of the development of the technology itself when using the Information and Communication Technologies (ICT). Therefore, the objective of this article is to review the main uses of the Semantic Web tools and to group them together in order to be able to propose the design and construction of more personalized and flexible systems, which allows to help people with disabilities to perform some type of activity using ICT, in this way, knowledge can be modeled in different domains related to people who have some type of disability, using ontologies, and some ontological models can be reused for various types of disability depending on the case study. The usefulness of this study is to reveal that with the models presented, it is possible to construct a Meta ontology that includes some or all areas of disability.
\end{abstract}

Keywords: ontology; disability; Semantic Web

\section{Introduction}

The size of the World Wide Web (WWW) has grown dramatically in recent years, which has led to a notable increase in the difficulty of finding more interesting resources about a particular topic, due in large part to ambiguity of the terms used in the queries that search tools use. The Semantic Web, sometimes also called Web 3.0 [1], aims to address the resolution of this problem, creating a mechanism for exchanging information, by adding a certain semantics or meaning to the existing Web, thus allowing establish a mechanism for automatic processing of your semantic content by a computer. To provide the Web with a meaning understandable by computers, it is necessary to have a certain way of representing knowledge. Therefore, as a solution for the distributed representation of knowledge, the Semantic Web proposes the use of information collections known as ontologies.

In philosophy, an ontology is a theory about the nature of existence and the types of things that exist. However, researchers in Artificial Intelligence have incorporated this term into their particular use with the meaning of a document or file that formally defines a set of concepts that are organized hierarchically, and that establishes the properties and relationships that exist between them, to which adds a set of inferences rules that allow to automatically manipulate the information.

Since there is a percentage of almost $15 \%$ of disabled people in the world [2] it is required that many of these people be inserted into society and in some cases Information and Communication Technologies (ICT) could help in this, but one of The problems that currently exist is that of the technological barrier that in many cases prevents its inclusion in society. Where social inclusion means integrating all members of society into community life, regardless of their origin, their activity, their socio-economic status or their thinking. Normally, social inclusion is related to the humblest sectors, but it may also have to do with discriminated and neglected minorities. 
According to the International Classification of Functioning, Disability and Health (ICF), disability is a generic term that encompasses deficiencies, activity limitations and restrictions on the participation of people [2]. In turn, accessibility is the degree to which all people can use an object, visit a place or access a service, regardless of their technical, cognitive or physical capabilities. It is indispensable and essential, since it is a necessary condition for the participation of all people regardless of the possible functional limitations, they may have.

At present there are several works that relate the use of ontologies in different domains and one of them is the relationship that is presented with disabled people, this has motivated our study and deserves a special analysis. In our final approach, we conducted a comparative evaluation of the cases studied that are from heterogeneous domains. However, several projects have developed some interfaces and ontologies such as: AEGIS Ontology [3], Rat Genome Database Disease Ontology [4], GUMO [5].

Therefore, the present work is divided as follows, in section 2 we make a literary review of the Semantic Web tools and their relationship with various types of disabilities applied in specific jobs, in section 3 we describe the most recent ontological models related to people with some type of disability, and in section 4 we make an evaluation of the systems studied. Finally, section 5 presents the conclusions obtained from the study carried out.

\section{Review of the literature related to the subject}

The research will be carried out based on articles related to the technologies of the Semantic Web and its relationship with people who have some type of disability. The bibliographic search was carried out in different electronic bases. In this section we will review the efforts made on the proposed theme from the year 2002.

In Zinnikus, Bogdanovich and Schafer proposed a recommendation system based on ontologies for the elderly and who also have some type of disability, is the basis of the SAID project that was funded by the Information Society Program of the European Commission (IST), to reduce complexity In this case, this is presented in a hierarchical tree [6].

Then in Bagues, Bermudez, Illarramendi, Tablado and Goñi use the ontologies in the development of an Innovation System for the Elderly called Telecare, which presents a new feature incorporated such as quality control, anywhere and anytime, in this work two ontologies were developed, one describes the domain knowledge and the other describes the system operation model [7]. Continuing with it Yesilada, Harper, Goble and Stevens introduced a semi-automated tool that supports travel and mobility of visually impaired users. This tool analyzes existing web pages, through the use of a travel ontology, in order to extract information that may be relevant to the user. This improves the browsing capacity of users with visual impairment [8].

In Schwinger, Retschitzegger, Puhretmair and Nussbaum developed an ontology based on a personalization approach to support people with special needs in order to adapt the services of their family environment, they use an ontology that is the basis of the personalization architecture that the authors propose [9]. Then in Karim and Tjoa, proposed an ontology to improve user interaction for people with special needs. This allows to improve access to personal information systems so it is possible for developers of the user interface to adapt them to the needs of users [10]. Independently Rudner and Ronnberg proposed a functional ontology related to sign language and generate a working memory model that makes strong predictions about neurological representation through sensory and linguistic modalities [11].

Then in Alfano, Brunetti, De Pietro and Esposito proposed an approach based on ontologies for the classification of white matter in abnormal patients with multiple sclerosis, in this work they show their taxonomy and the rules they use, based on studies and results obtained in brain images obtained after applying the Magnetic Resonance to various patients of the hospital where the case was studied [12]. Regardless Latfi, Lefebvre and Descheneaux developed a management ontology, related to older people with the loss of cognitive autonomy. They mention that caring for an elderly person in the loss 
of cognitive autonomy is a difficult task and for this they use artificial agents in an intelligent system in the house called Telehealth (TSH) to facilitate this task [13]. Next Gennari and Di Mascio makes efforts to develop an ontology for an Italian sign language dictionary on the web, from this work Felice, Di Mascio and Gennari [14] proposed a visual interface of an Italian sign language dictionary on the web based on an ontology, they propose the prototype of a dictionary called e-LIS which was the first two-way web in Italian sign language [15].

In Pfalzgraf, Pfleger, Schehl and Steigner proposed a Dialogue Platform based on ontologies that they call ODP, this platform will be used in some interfaces of other projects [16]. Otherwise Keet, Alberts, Gerber and Chimamiwa developed the improvement of web portals with an ontology based on Access to data and took the case of a study carried out in South Africa for the development of the Portal. For this they develop an ontology called ADOLENA with the OWL language [17].

Regardless Tang, De Baer, Zhao, Meersman and Pudkey developed an ontology modeling methodology for the care of the elderly in households in the United Kingdom, the methodology is implemented with the tool called PA-DON [18]. Then Lim, Tam, Wong, Liu and Lee implemented a web system for learning for all people, based on the integration of two ontologies, such as content-based learning and user-based learning [19].

In Kehagias and Tzovaras developed a framework based on ontologies for the integration of various web services and delivery to users with reduced mobility, this proposal serves people with reduced mobility to search the Web for any service they need. To access all the contents on the web, you must be equipped with the tools of the Semantic Web, this work has been developed in a project called ASK-IT presented in Europe [20]. Then Zakraoui and Zagler developed an ontology called CO to represent in context user interaction and improve accessibility to the web for all people. In this work, 4 important concepts are identified such as: user context, physical context, environmental context and computational context [21].

From an earlier work Nganji, Brayshaw and Tompsett developed an ontology called ADOOLES based on personalized online teaching for disabled students in higher education, this is due to the increase in students that occurs every year in the United Kingdom. This ontology is based on another called ADOLENA [22]. Then Gupta and Garg developed a prototype system based on an ontology for the recovery of internet information for autistic people through learning styles. Where the autistic user wishes to recover for example products and these may have certain different characteristics. However, this is difficult for autistic people, due to their lack of ability to process and retain information and therefore develop such an ontology [23].

Regardless of this Bukhari and Kim developed an automatic assisted ontology for precise extraction of information to help people with visual impairments. By increasing information on the internet, the extraction of information is becoming increasingly difficult and this poses a problem for people with visual disabilities, because these users require help and special tools for the extraction of relevant information through Internet. The ontology they propose is called HOIEV [24]. In Alonso, Aginako, Lozano and Olaizola presented a middleware based on ontologies to retrieve and classify information in places adapted for people with special needs, based on the PATRAC project. In addition, they propose a content manager based on ontologies in 3 designed modules, the manager uses SOAP (Simple Object Access Protocol) web services. The authors propose an innovative solution based on the ontology to customize tourism aimed at people with special needs [25].

Independently Vassilev et al. presented ONTOCARER which is an ontological framework to assist people with disabilities using intelligent agents, the authors use the International Classification of Functionality, Disability and Health (ICF) that has been established as a standard for the purpose of classifying the various states of health. The ontology whose domain is disability is the core of ONTOCARER [26].

Then, in Rivas et al. proposed a modular and accessible platform that provides a computing environment for people with disabilities accessible through control devices adapted to the television set in users' homes. In their work they show how semantic technologies can be applied to increase 
perceived accessibility and inclusion by reducing dependence on other people. In their work they create an intelligent tutor for the recommendation of work depending on the disability of a particular individual with the technologies of the Semantic Web [27]. In the work of Zerkouk, Calvacante, Mhaded, Boudy and Messabih mentioned that with the increasing proportion of dependent people (elderly, disabled users) in the world, telecare and televigilance platforms will play an important role in providing a Efficient and less expensive remote care in assistance environments. The authors use ontologies to model their personalized assistance system, because the data they obtain from various measurements made with multiple sensors are heterogeneous data [28].

Independently in Rosales, Castillo, Puente and Boza proposed a prototype of a semantic platform with web accessibility for people with visual disabilities. In their work they propose a method of interaction with the user through voice commands, allowing direct communication with the platform. The platform proposed by the authors will be implemented using tools from the Semantic Web [29].

In the work of Djaid, Dourlens, Saadia and Ramdane mentioned that the goal is to build a multimodal system that helps people with disabilities to interact with their environmental environment, using natural skills, such as voice and gestures. The proposed system uses fusion and fission engines and also contains an ontology where base knowledge is stored. This designed system is validated through case simulations [30]. Then, in Ali et al. mentioned that the capture and interpretation of the voice has generated the use of the so-called social robots to transmit information and provide recommendations. However, because search engines are based on keywords, they are inadequate to deliver relevant information from queries made. From this, the authors propose a combined system of extraction and recommendation of information based on vector support machines to which an ontology for people with disabilities is merged [31]. In Fleiner, Szász, Nagy and Micsik presented a data model in the form of an ontology that includes the description of the indoor location of the hospitals, the indoor navigation characteristics and the accessibility attributes to help people with movement disabilities. In their work, the authors use 2 ontologies and use Semantic Web technologies such as: RDF, OWL definitions and SPARQL queries for the navigation functions of their applications [32]. Independently in Torres, Rodriguez, Espin and Visitacion mentioned that people with special needs may have difficulties accessing and using mobile applications. From this, the authors present in their work a new classification of user disabilities that implicitly build user profiles based on ontologies, which can be used by recommendation system. A complete user profile could provide better accessibility to new technologies for users with any disability, which would lead to greater inclusion [33].

Finally, in Fattah and Chong mentioned that recent advances in medicine have made people live longer, which has affected in many aspects, such as the increase in the cost of medical care, the shortage of caregivers, the number of disabled people with depressive disorders, etc. From this, researchers are interested in assistance services for the elderly and disabled in smart home environments, which currently contain many smart devices and are interconnected in the network environment and are based on the Internet of Things (IoT). The authors then propose a Web services framework called RESTFUL, which uses an ontology to create assistance services for older people in smart home environments using the so-called Object Web (WoO) [34].

\section{Ontological models related to people with disabilities}

In this section, the ontological models that relate different types of disabilities from 2010 to 2018 will be analyzed more broadly.

\subsection{An ontology-based framework for web service integration and delivery to mobility impaired users}

In Kehagias and Tzovaras, 2010 [20] they observe the needs of users with reduced mobility, while they are planning to make a trip from one city to another, or carrying out their control activities at home during the trip, from this they present a work that describes a framework based on ontologies, whose purpose is to collect the content of several existing Web services and obtain accurate information 
on these needs to help elderly users and people with disabilities. In order to access all the content required by the user, the tool is equipped with tools from the Semantic Web of services search and discovery mechanisms.

The present work was developed in the project called ASK-IT, presented in Europe, whose objective was to develop an intelligent environment with a framework that supports the needs of users with reduced mobility when traveling from one place to another. ASK-IT ontologies are classified according to the following domains:

- Services Ontology. This ontology includes the description about support services.

- Transportation Ontology. This ontology includes information related to transport and different types of data, which can be filled out by content providers and serves people with reduced mobility.

- Ontology for tourism and Vacations. This ontology contains what is related to tourism, that is, it would interest a tourist when visiting an area or city. This includes, among others, useful content about hotels, museums, places of interest, as well as information on tourist offices, embassies, airlines, telephone numbers and useful addresses.

- Support Service Ontology. This ontology includes information about fellow travelers, specific attention at the hotel, eating, paramedical assistance, etc. These services will be used by people seeking assistance and their needs (including languages, experience, specific preferences).

- Ontology e-learning and e-working. It includes concepts based on work and education services on online teaching with special considerations for users with reduced mobility.

- Ontology of social relations and community building. This ontology contains information about social relationships, community registration and social events.

Part of the taxonomy is presented in the figure 1:

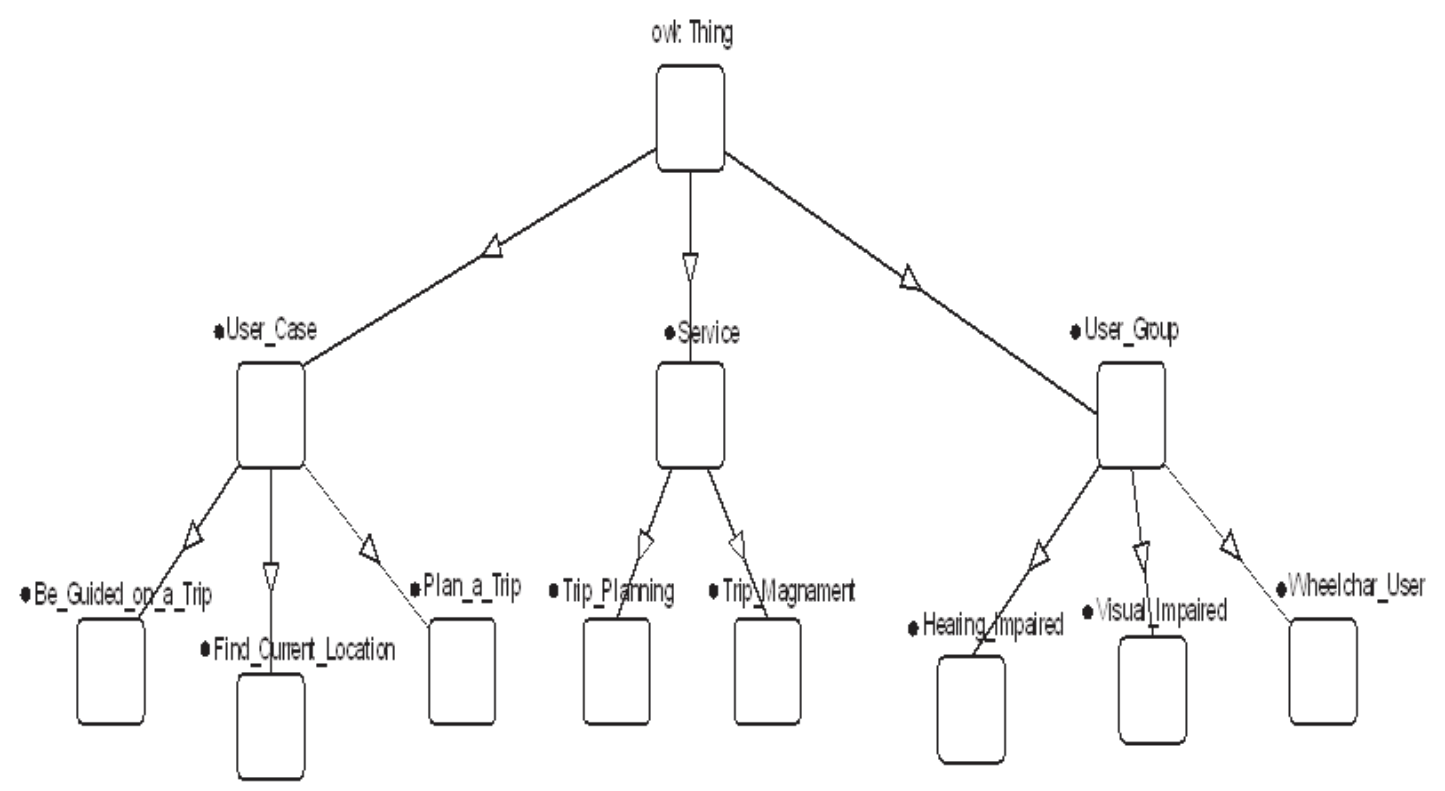

Figure 1. Taxonomy of Ontology (Kegahias and Tzovaras)

\subsection{An ontology for representing context in user interaction for enhancing web accessibility for all}

In Zakraoui and Zagler, 2010 [21] they observe that the majority of online educational environments are not accessible to all users, such as e-learning systems, which in recent years has become popular as an educational tool that offers a great opportunity for learning, the authors observe that there are many electronic barriers that prevent access to online resources and present an ontology 
to represent user interaction in the context and improve accessibility to the Web of all people and they call it $\mathrm{CO}$ (context Ontology).

The authors consider that this ontological model will improve interaction with the user, in the proposed ontology all the factors that could influence Web accessibility, such as relevant environmental parameters (noise, light, etc.) are considered, there are also other parameters that can use: location, presence of objects or people, temperature, blood pressure of the user, etc. In fact, any environmental factor that can influence user interaction can be used, provided there is some mechanism to capture it.

For the construction of the ontology the authors consider all the factors that can influence the Web accessibility of people, such as if a person with visual impairment wants to use their mobile device to connect to the e-learning platform, in said ontology I would find that context factors would help the person to achieve this. The figure 2 shows part of the taxonomy of the case.

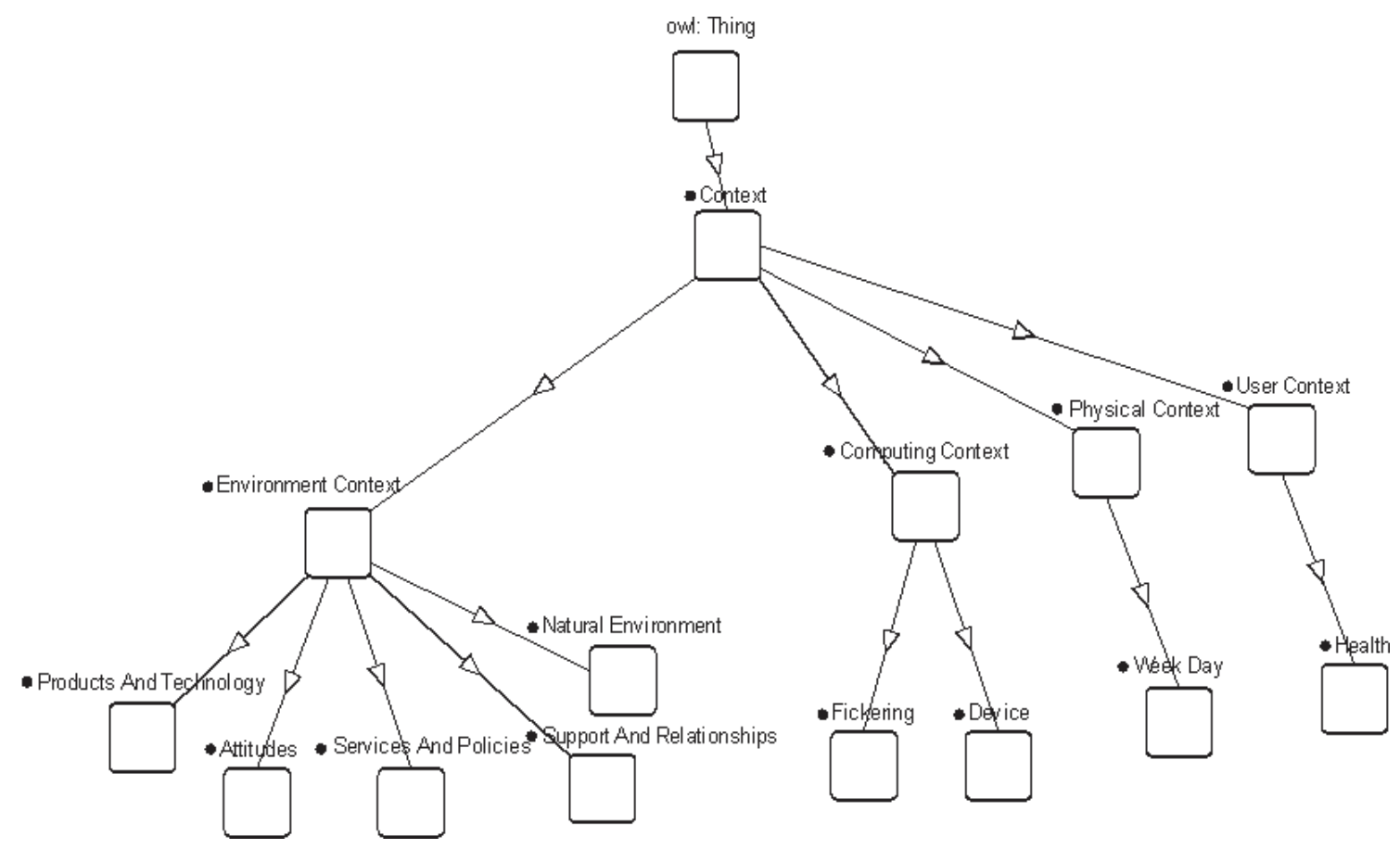

Figure 2. Taxonomy of Ontology (Zakraoui and Zagler)

\subsection{Ontology-based e-learning personalization for disabled students in higher education}

In Nganji, Brayshaw and Tompsett, 2011 [22] they base their work on the basis of the following information they acquire in the United Kingdom:

- The number of students with disabilities in higher education institutions increases every year.

- The use of online education is increasingly difficult, as institutions encounter students with some type of disability that require adjustments to learning environments.

- The law requires that people with disabilities will receive the same learning experiences as their non-disabled peers through reasonable adjustments.

Faced with these problems, educational institutions are using assistive technologies to help students with disabilities in learning, but some of these technologies are incompatible with some learning environments, thus excluding some students with disabilities. To solve this problem, the authors propose the customization of online teaching and this is achieved using the technologies of the Semantic Web such as the use of ontologies. Where knowledge is modeled on an ontological model, where the learning of students with disabilities in higher education is customized.

The ontology has concepts related to disability, assistance mechanisms and services, and other types of classes. The proposed ontology is called ADOOLES. It incorporates some of the concepts 
contained in the ADOLENA ontology developed and used by Keet, Alberts, Gerber and Chimamiwa, 2008 [17] to improve the National Accessibility Portal (NAP) and also HESA (Higher Education Statistics Agency) concepts related to the Disabilities have been incorporated, the ADOOLES ontology has vital information regarding various types of disability found in UK higher education.

The authors also propose an architecture based on the ontology, for the construction of an e-learning system for students with disabilities in higher education in the United Kingdom. Finally, the e-learning system that the authors present helps solve some related problems encountered by students with disabilities in higher education, particularly the inaccessibility of learning and environments incompatible with technological assistance. Part of the taxonomy of the case is presented in the figure 3 .

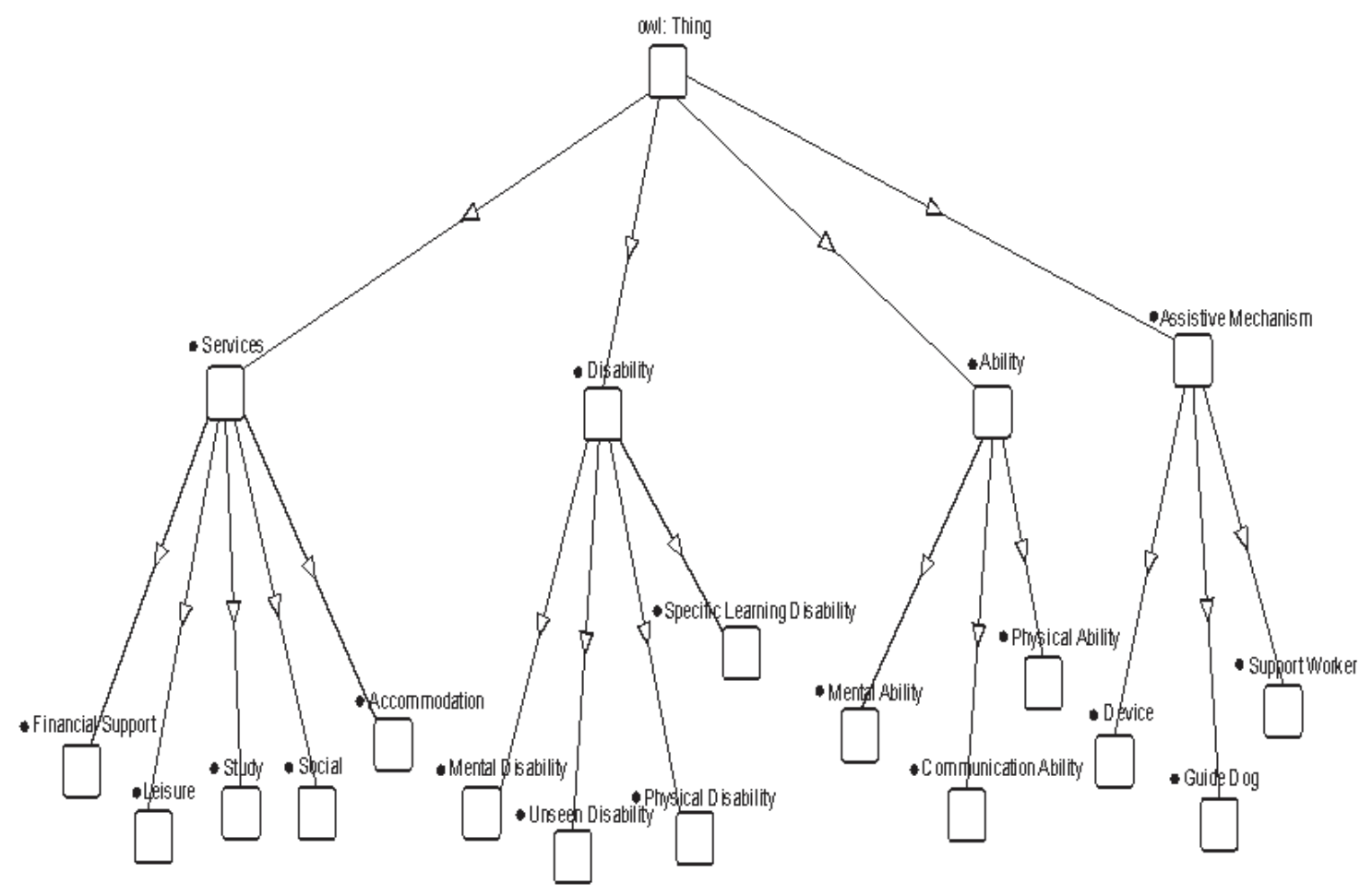

Figure 3. Taxonomy of Ontology (Nganji, Brayshaw and Tompsett)

\subsection{Ontology based information retrieval for learning styles of autistic people}

In Gupta and Garg, 2011 [23] they observe that the autistic user wishes to recover certain products that have certain characteristics such as: size, length, color and other parameters that they need. However, this is difficult to do for this type of users, mainly due to their ability to process and retain information. The authors present a design of a prototype system for keyword-based queries, and use an ontology to retrieve information from the internet, and help these types of users identify these characteristics.

The objective of the work is that the user can retrieve that information looking for the right set of keywords based on the memories that these people have. One of the most notable mysteries of autism has been the ability of most autistic people to have spatial visual skills, that allows them to build complete systems in their imagination. They prefer to use diagrams, photographs and movies to see the information they are learning. Then for the autistic person to remember something, he must remember when and where the incident occurred and then identify it. Research has also found that students with autism who use computers have increased their attention spans, and can remain in their seats for quite some time, in addition to developing better fine motor skills. In their ontology they present a diversity of games to help the learning of these children, the following figure shows 
part of the taxonomy of the mentioned case. The authors also present a possible architecture for their prototype system. Part of the taxonomy of the case is presented in the figure 4

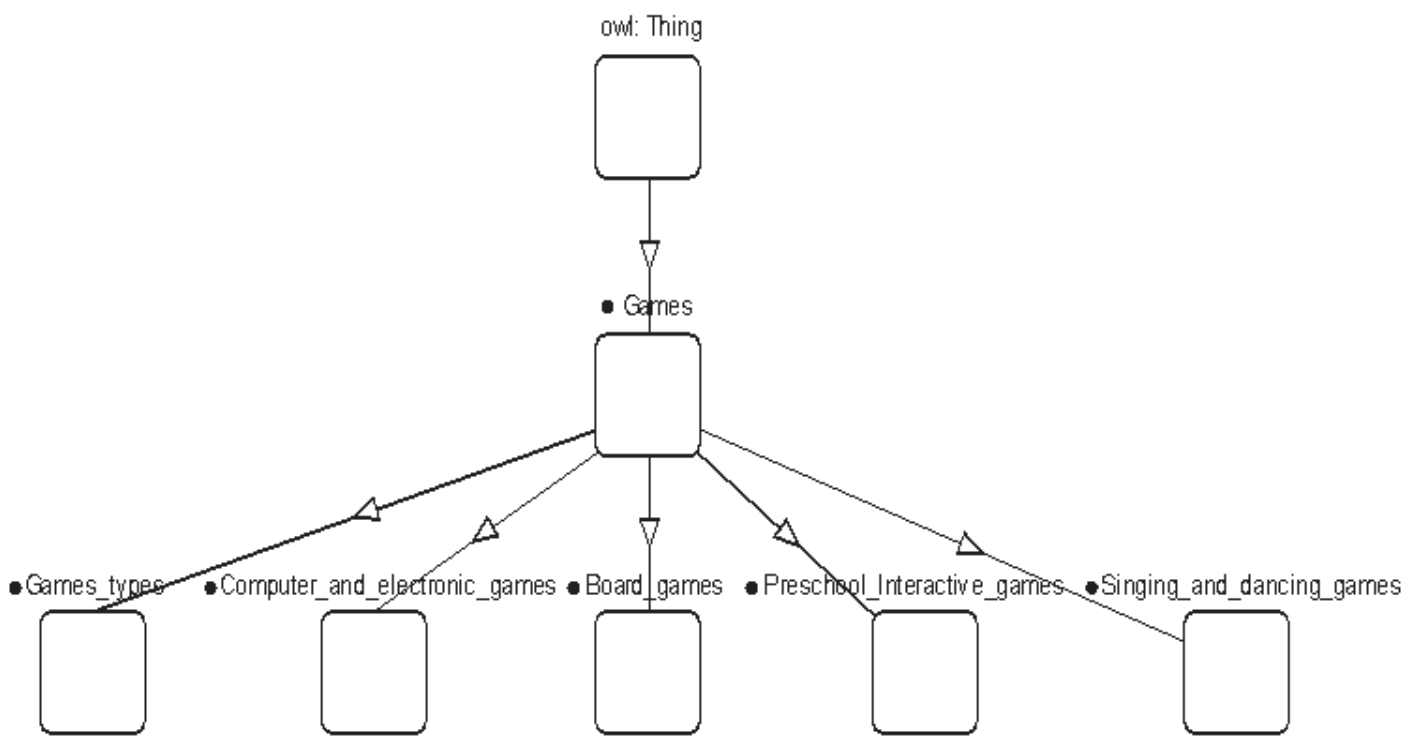

Figure 4. Taxonomy of Ontology (Gupta and Garg)

\subsection{Ontology-assisted automatic precise information extractor for visually impaired inhabitants}

As the Internet grows rapidly, millions of Web pages are added to the servers and the extraction of accurate information is becoming more and more difficult, as the volume of data dramatically increases on the Internet. While there are several search engines and tools for searching for information that are available on the Internet. For the most part, these search engines are based on keywords. This causes a problem for visually impaired people who wish to obtain full use of available online resources. Users with visual disabilities require some special help to work well with any computer system, such as a certain interface and a content manager, in addition special tools are required to facilitate the extraction of relevant information through the Internet for users with this kind of problem.

In Bukhari and Kim, 2012 [24] they observe the problems that people with visual disabilities have to obtain information on the computer, they propose the ontology called HOIEV (Ontology based on the extraction of information for people with visual disabilities), where the proposed architecture provides a mechanism for extracting high precision information from the ontology and one using a voice command system for internet users with visual impairment. For their communication users use a Smartphone with Pocket CMU SPHINX-2 installed as an application on the phone. The HOIEV consists of 3 layers: interaction with the remote user, extraction of information from the core and the programming interface (API). The intelligent prototype system communicates with the different tools, such as voice command analyzers, ontology domain information extractors and a short message engine, finally introducing an autonomous information extraction mechanism. The figure 5 shows part of the taxonomy of the case. 


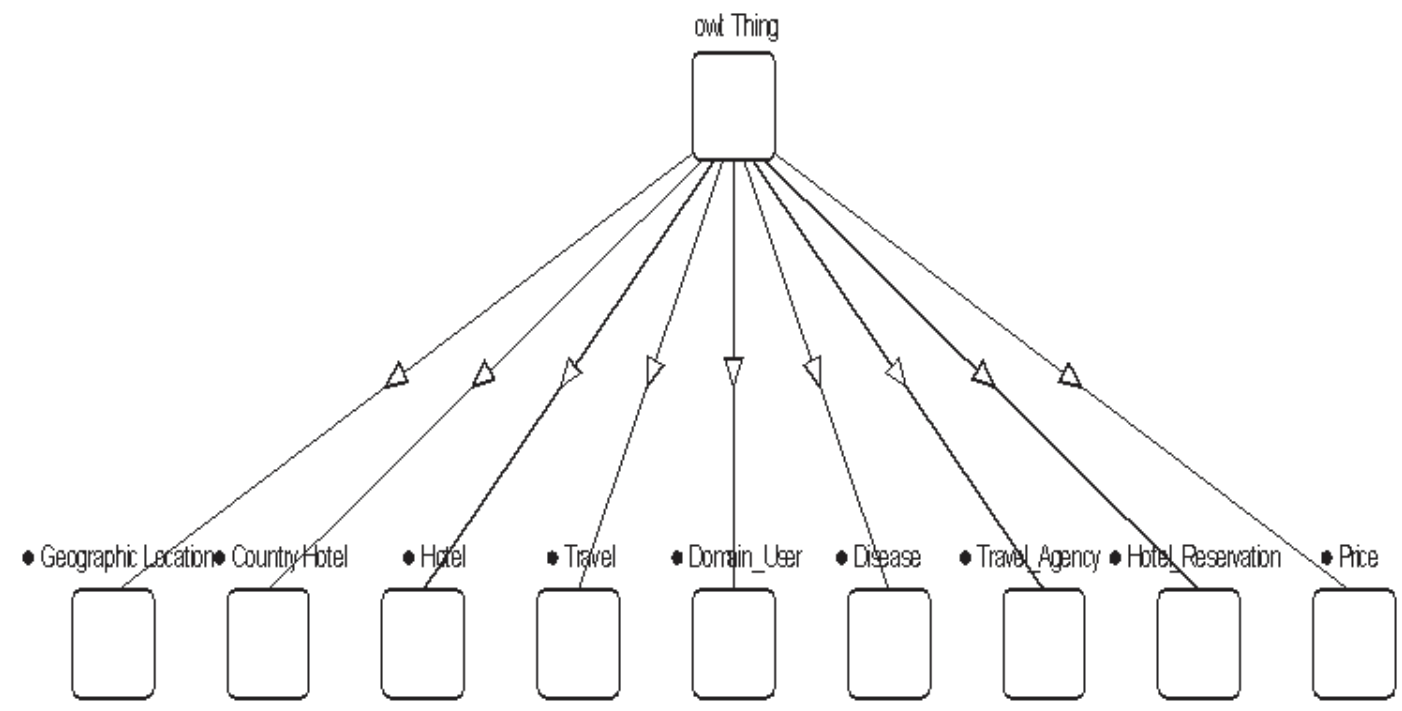

Figure 5. Taxonomy of Ontology (Bukhari and Kim)

3.6. Ontology based middleware for ranking and retrieving information on locations adapted for people with special needs

In Alonso, Aginako, Lozano and Olaizola, 2012 [25] they observe that the current search tools for tourist services do not take into account the special needs of many people with diverse functionalities, they propose a solution based on the different technologies of the Web Semantics, which would achieve more personalized searches and propose an ontology for personalized tourism aimed at people with special needs.

The work is based on the philosophy of the PATRAC project (Accessible Heritage for a culture without barriers) where an ontological model is used for the evaluation of accessibility in cultural heritage environments. The proposed work has been implemented in a software module, and is integrated into an interactive visualization service for the community, this module offers a greater search capacity to help all types of users in the search for a special type of restaurant.

The proposed system consists of several modules and one of them is the content manager. The functionalities and implementation details of the content manager are shown in the figure 6.

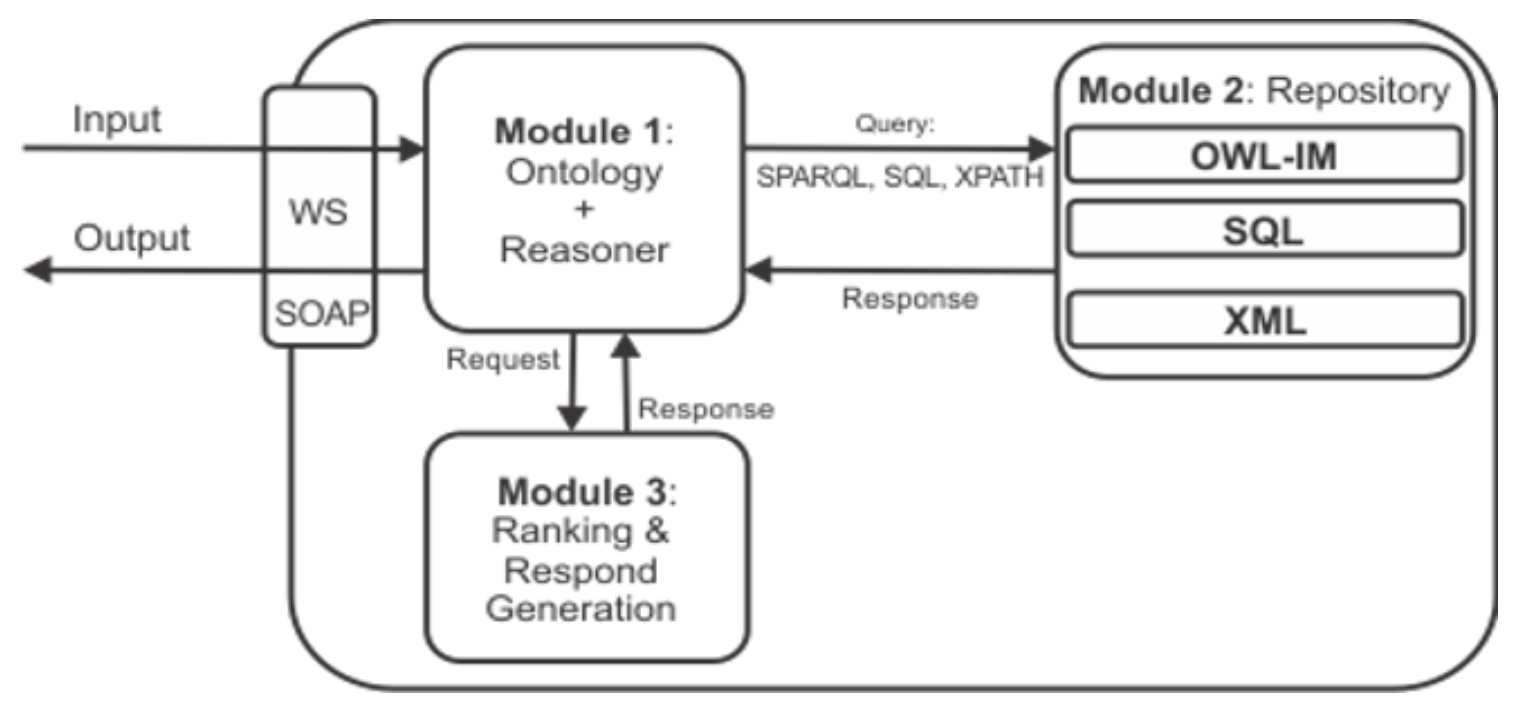

Figure 6. Content Manager (Alonso, Aginako, Lozano and Olaizola)

The input and output of the content manager are based on Web Services SOAP (Simple Object Access Protocol), where the service request made to the system can be divided into two categories. 
The first one is related to general preferences such as: real search parameters and general parameters of the person's profile. The second is related to context preferences such as: type of disability and gastronomic needs. The content manager consists of three different modules:

- $\quad$ Module 1: Ontology and reasoning.

- Module 2: From repository.

- Module 3: Classification and Generation of Responses.

An example that summarizes the content manager functionalities is the case of deaf people looking for a restaurant. Since the system is aware of your deafness, restaurants with round tables and a staff that knows the language of signs that allow offering communication services for deaf people will be sought. On the one hand, round tables will allow deaf people to observe everyone at the table, and they can also read their lips more easily. The output of the content manager will first show the restaurants that best fit the user's request in their preferences and personalized needs.

\subsection{OntoCarer: An ontological framework for assistive agents for the disabled}

People with disabilities have different needs based on their specific disabilities and the assistance provided to them should be tailored to their needs. In Vassilev et al. 2013 [26] they contribute the so-called OntoCarer which is the framework of a research program of the Center for Intelligent Systems Research of the Metropolitan University of London, whose objective is to use technologies that help older people and people with disabilities to the use of the Web and mobile Internet. The authors propose an ontology whose domain is the disability that is the core of the framework of the so-called OntoCarer. Within this framework, software agents will be used that will act in favor of both assistance and assistance to people. Its conceptual basis is based on what the World Health Organization (WHO) states, for the help of people with disabilities.

The OntoCarer program, to make the solution accessible uses only standard communication infrastructure such as (internet and mobile internet), public hosting service (Web servers), free software tools (databases, repositories and libraries) and cheap mobile equipment (Android smartphones and PDAs). In this project the software agents will help the person attending and the person assisted, these software agents will be placed on the mobile phones, and coordinating through another agent, the different activities to be performed.

The authors do not create a new ontology, but use the one developed in the International Classification of Functionality, Disability and Health (ICF) that has been established as a standard for the purpose of classifying the various states of health. This Ontology currently consists of approximately 1500 classes and there are all possible cases of disability. The structure of the ICF is organized in 2 parts each with 2 components:

- Part 1: Functioning and disability. Its first component is constituted of bodily functioning and structures and the second component is constituted of activities and participation.

- Part 2: Contextual factors. It consists of environmental factors and personal factors.

An example of the taxonomy partially is shown in the figure 7. 


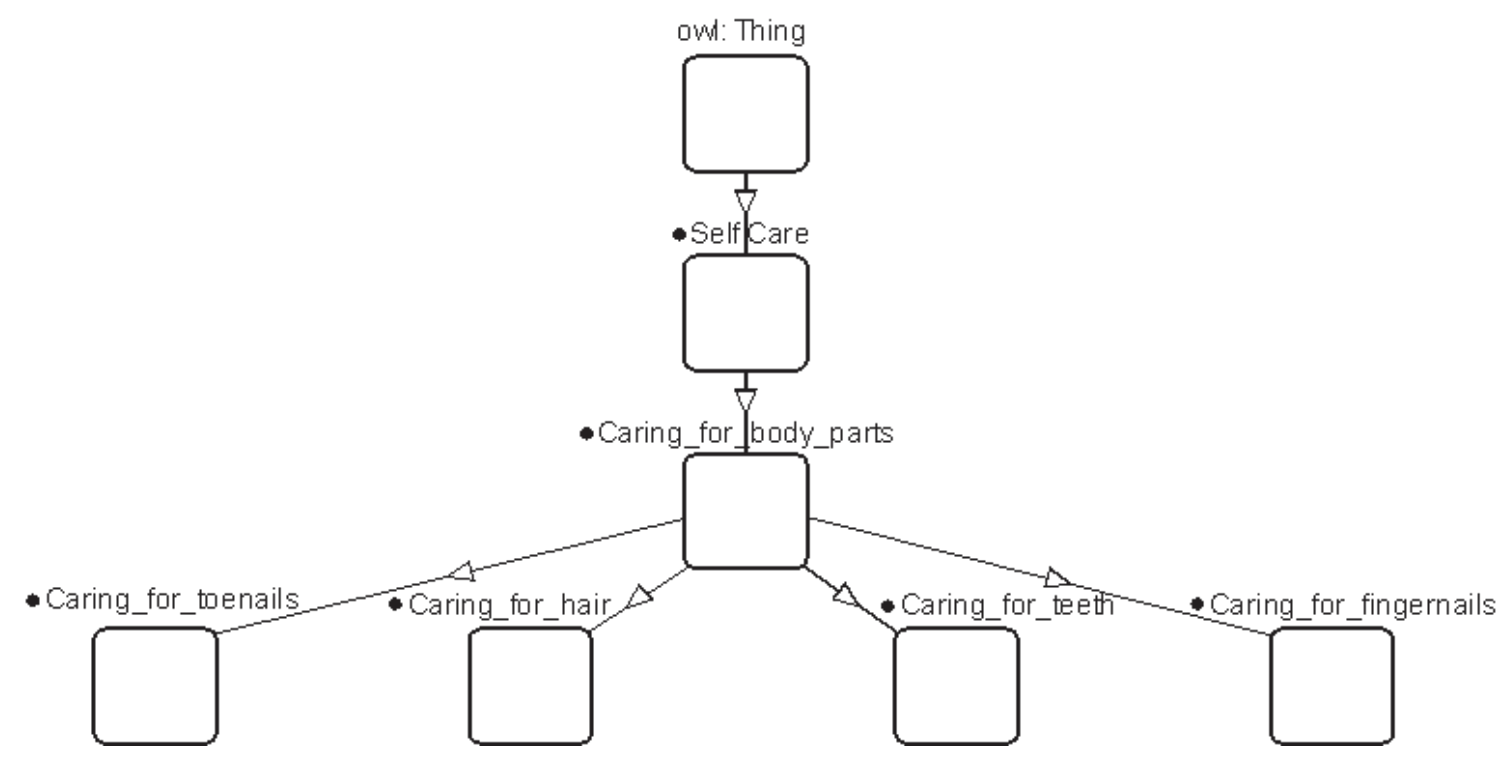

Figure 7. Taxonomy of Ontology (Vassilev et al.)

\subsection{An Accessible Platform for people with disabilities}

In Rivas et al. 2014 [27] they propose an accessible modular platform that provides a computing environment for people with disabilities through control devices adapted to the television set in the users' homes. Relying on a standard television set, which is the device that allows dependents, especially people with disabilities, to introduce new technological advances in communication and information management in their daily lives. The solution presented by the authors offers a set of educational and training services related to teleworking and job search aimed at people with a particular disability.

To facilitate the construction of the knowledge model, the authors propose a semantic model that falls within the domain of the personalized work recommendation for people with a certain degree of disability. The developed system also maintains information about user profiles and also work information describing offers published in a digital medium. The recommendations on the most suitable job offers will be generated according to the profile of the users registered on the platform. The architecture of the platform proposed by the authors is based on a service-oriented architecture (SOA). The functionalities developed are encapsulated as distributed web services that communicate with each other through standard technologies such as SOAP (Simple Object Access Protocol), WSDL (Web Services Description Language), UDDI (Universal Description Discovery and Integration).

In their work, the authors also show how semantic technologies can be applied to increase accessibility and inclusion by reducing dependence on other people's help. The platform developed allows the integration of semantic technologies, interfaces and various adapted devices. This integration process will simplify the task of finding jobs suitable for the needs and characteristics of users with disabilities. The conceptualization scheme of its model is shown in the figure 8 . 


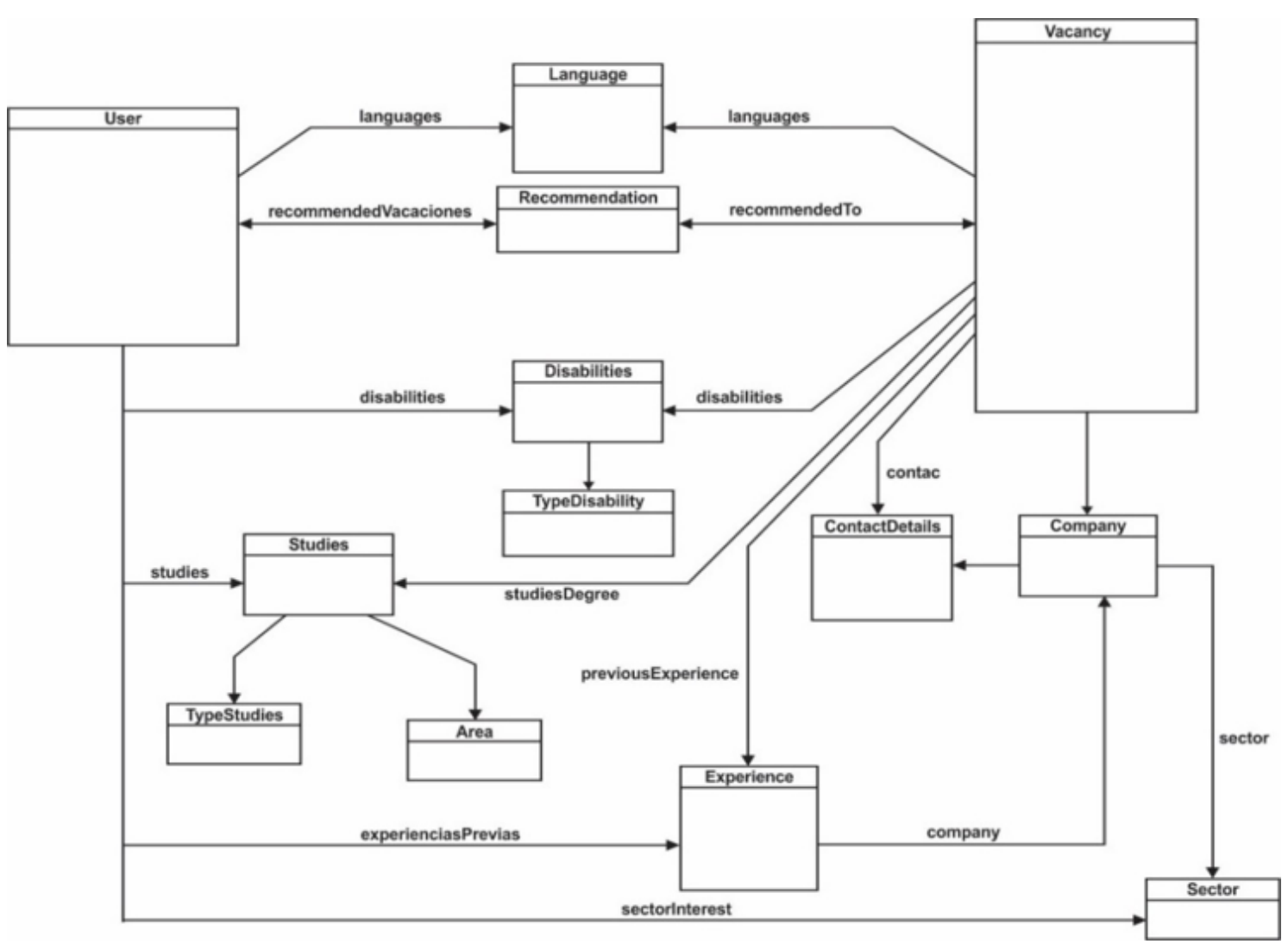

Figure 8. The Conceptual model (Rivas et al.)

\subsection{Behavior and Capability based Access Control Model for personalized TelehealthCare assistance}

In Zerkouk, Cacalvante, Mhaded, Boudy and Messabih, 2014 [28] they mention that with the growth of dependent people (elderly, disabled users) in the world, telecare and televigilance platforms will play an important role in providing efficient and less remote care expensive in your support environments. Where the technology used based on sensors, would contribute greatly to obtain valuable information that should help provide personalized access to the services available within their living spaces. However, current access control models remain inadequate due to certain factors such as lack of integrity, flexibility and adaptability to the user's profile. Based on this, the authors propose a new access control model based on the user's capabilities and behavior. To implement its model, it uses three main mechanisms such as: Acquisition, management and security.

The acquisition of context data is done through a televigilance platform used by the authors, for management it focuses on the modeling and reasoning process that aims to manage the collected context, this model is based on a dynamic ontology and Security is done through consultation processes that evolve with the access rules implemented in the model.

As the data obtained with sensors are complex and heterogeneous and are related, for example, to posture, movement, falls, time, user presence, emergency calls, etc. That is why authors to model knowledge use ontologies, since these are more appropriate to model these contextual data. According to the authors, the designed ontology is chosen as the appropriate means to use it as a system security policy. The proposed ontology is defined through semantic web technologies, the ontology language (OWL), the rules language (SWRL), the RDF and the query language (SPARQL). Next, the following figure shows the ontological security model developed with the Protegé editor, the classes and subclasses implemented are observed. As classes the authors define: the user, the device, the service, the environment and the security policies. Part of the ontology is shown in the figure 9 . 


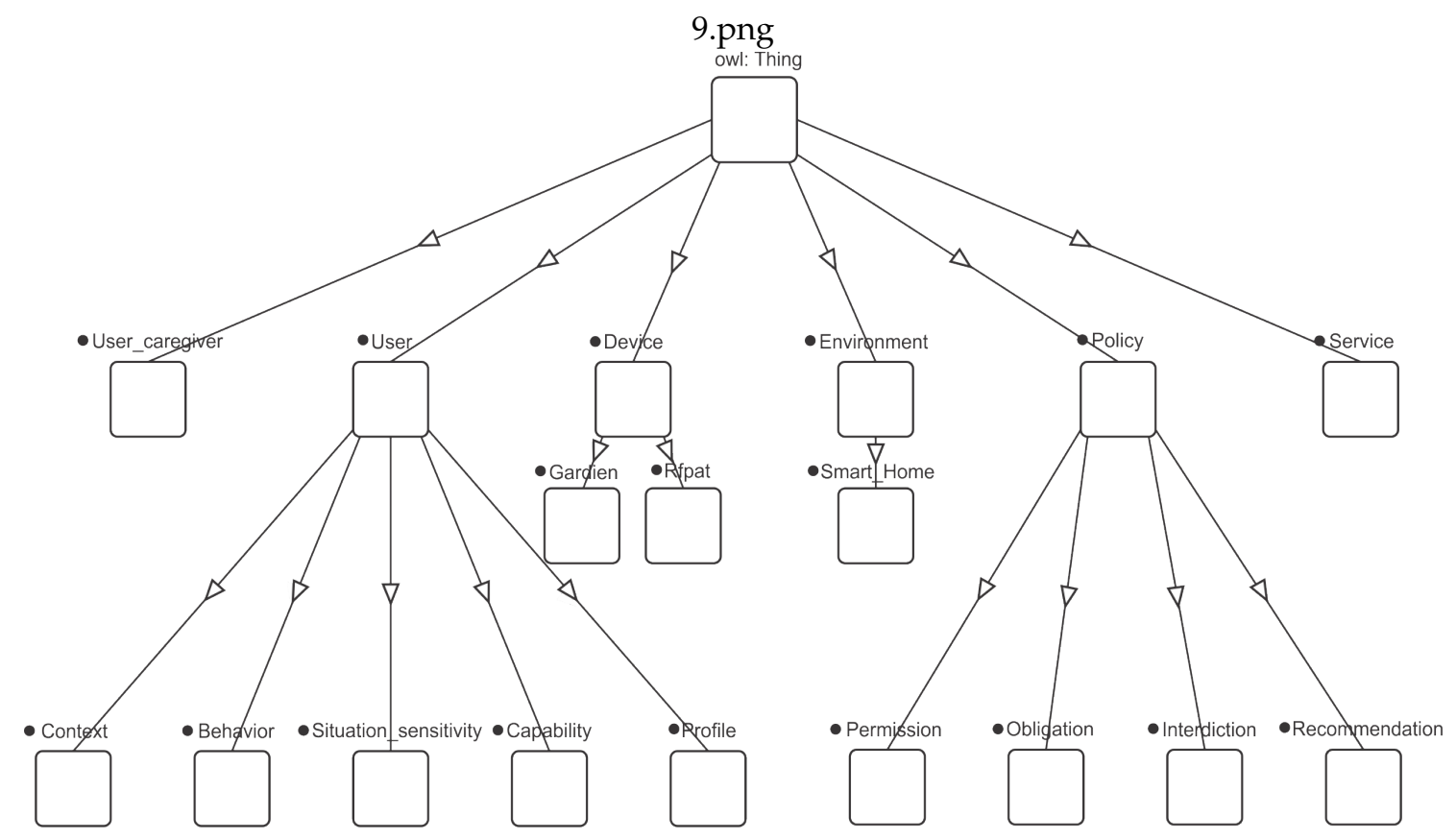

Figure 9. Ontology of security policy (Zerkouk, Cacalvante, Mhaded, Boudy and Messabih)

\subsection{A Prototype of a Semantic Platform with a Speech Recognition System for Visual Impaired People}

In Rosales, Castillo, Puente and Boza, 2015 [29] they mention that a percentage of the world's population suffers from some type of disability, where rapid technological development can cause some barriers that these people must face if they want to access technology. In the case of visually impaired users, special assistance is required when using any computer system and also depends on the audio for navigation tasks. The authors focus on making a prototype of a semantic platform with Web accessibility for people with visual disabilities.

They propose a method of interaction with the user through voice commands, allowing direct communication with the platform. The proposed platform was implemented using Semantic Web tools, because it facilitates the search and retrieval of information in a more efficient way than the current web. In addition, the Google STT APIs (Speech To Text), TTS (Text To Speech) and the Raspberry Pi board are integrated into the voice recognition module.

As a domain of ontology, the authors use the domain of tourism and mention that, in their country, tourism is a sector of considerable growth. Then traveling involves multiple needs such as: accommodation, food, transportation, etc. From this, the tourist with visual disability needs to know the various sources of information related to tourism using the developed prototype can find the required information to the domain of tourism.

For example, if the tourist consults by voice "Find 3 star hotels in the city of Ica", the prototype developed by the authors returns the answer indicating all the hotels that meet that characteristic. The ontology is show in figure 10. 


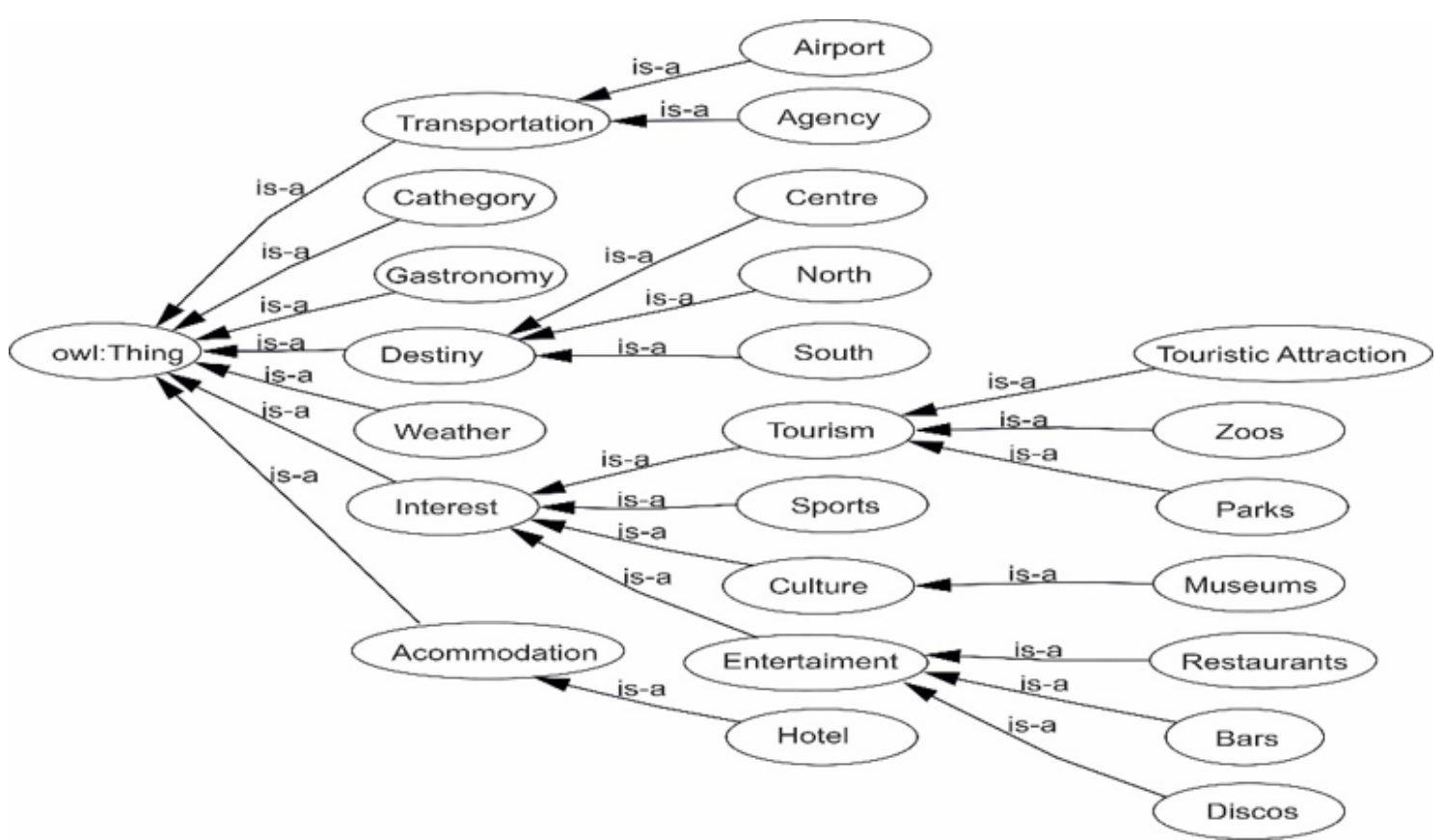

Figure 10. Ontology of Tourism (Rosales, Castillo, Puente and Boza)

\subsection{Fusion and fission engine for an assistant robot using an ontology knowledge base}

In the work of Djaid, Dourlens, Saadia and Ramdane, 2017 [30], they propose a multimodal system that helps people with disabilities to interact in intelligent environments, using their communication skills, such as voice and gesture. The authors present a new solution to facilitate human-machine interaction by building a multimodal intelligent system that uses fusion and fission processes. The combination of these two processes ensures that the input mode is understood and the action corresponding to the given situation is implemented. In addition, they use the semantic representation of the environment to facilitate the interpretation of fusion and fission processes. This ensures a common and reliable representation of the data exchanged between the user and the machine, which means that the data has the same format and meaning for both the machine and humans.

In the architecture proposed by the authors, they use fusion engines, fission and a knowledge base known as ontology. To initiate queries to the system, the wheelchair user can use multimodal inputs, such as his voice, gestures, etc. To request certain types of services, then the system detects the inputs of the environment using sensors and merges them using the fusion engine to understand their meaning. Then subdivide the necessary action (depending on the situation deduced from the fusion engine) using the fusion engine and send unique tasks to generate actuators or devices and execute the action corresponding to the user's request, using fission engines. Both fusion and fission engines depend on the ontology, which is the knowledge base of our system, where the contextual semantic information designed by the authors is located.

The ontology created allows to take into account a large number of elements that describe the user's environment. In addition, it allows the integration of rules that must be taken into account during the selection of the modality, as well as in the merger and merger process. Next, a part of the ontological model is shown and the Environment class (a superclass) that contains various entities around its environment is described as:

- Input mode: contains all the modalities used in the system: they can be speech, gesture, look, manual mode (the keyboard and mouse of a computer) and touch.

- Output mode: it consists of moving output, visual output and vocal output.

- Alarm: contains the alarms used by the system and can be a system or health alarm.

- Surroundings: it consists of all the elements present in the user's environment. These can be people, objects and places that surround the user. 
- Vocabulary: contains the words used when a user requests a service.

- Time: is the maximum time allowed for a command to be accepted and the maximum time between two successive modes.

- Context: it consists of the context of the environment, the context of health, the context of the user and the context of the system. For example, the context of the environment takes into account the elements that can affect the modalities, such as the level of lighting, the noise level, the climatic conditions and the ambient temperature.

- Fusion and fission model patterns: they contain our predefined models of different commands and solutions used in fusion and fusion engines, respectively.

Part of the ontology, the Environment class, is shown in the figure 11.

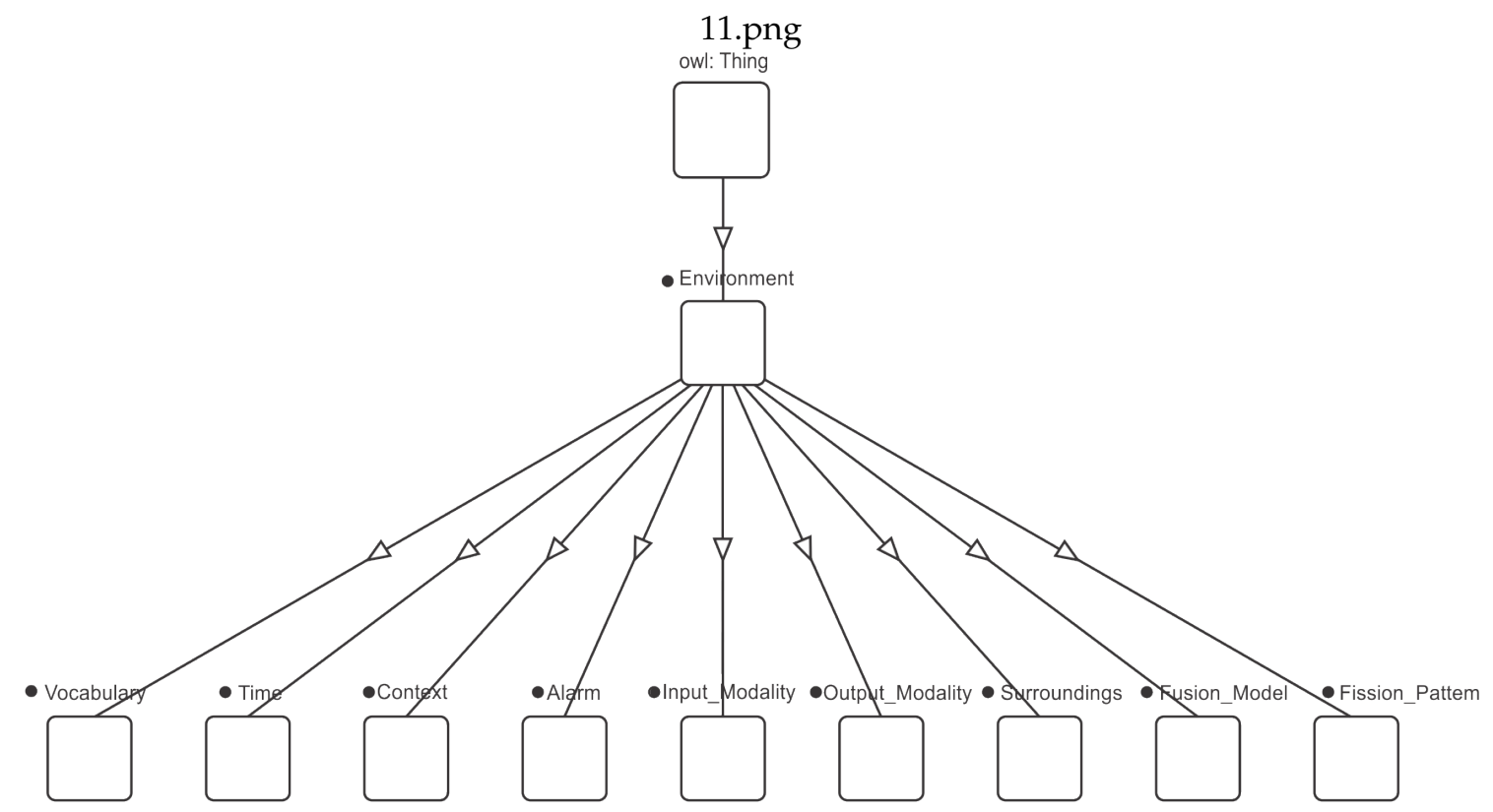

Figure 11. Environment Class (Djaid, Dourlens, Saadia and Ramdane)

\subsection{Merged ontology and SVM-based information extraction and recommendation system for social robots}

In the work of Ali et al. 2017 [31] they mention how recent technology for capturing and interpreting the human voice has generated the use of social robots to transmit information and provide recommendations. This technology helps people obtain information on a particular subject after an oral consultation with a humanoid robot. However, most current search engines are based on keyword search mechanisms, then existing full-text search engines are inadequate to retrieve relevant information. of several oral consultations. With only predefined words and recommendations based on sentences, a social robot may not suggest the correct elements, if the elements recovered along with the information are not predefined. In addition, the available conventional systems cannot extract accurate data from the web pages to show the correct results. From this, the authors propose a combined system of extraction and recommendation of information based on Vector Support Machines (SVM) to which an ontology is fused where the knowledge base is found. In the proposed system, when a humanoid robot receives an oral query from a disabled user, the oral query changes to a full-text query, the system extracts the full-text query to extract the needs of the disabled user and then converts the query into The correct format for a search engine.

The proposed system downloads a collection of information on items (for example, city characteristics, diabetes medications and hotel characteristics). The SVM identifies the relevant information about the query and removes anything irrelevant. A comparison analysis is used in the merged ontology to find the similarity of the element for a good recommendation for the user with disabilities. 
The ontology fused in his work, is a combination of 4 ontologies from various domains, such as: hotels, cities, transport and medicine. Below is part of the ontology, the hotel class, which provides semantic knowledge to extract information from the Internet related to the hotel and its characteristics. Part of the ontology, the Hotel class, is shown in the figure 12.

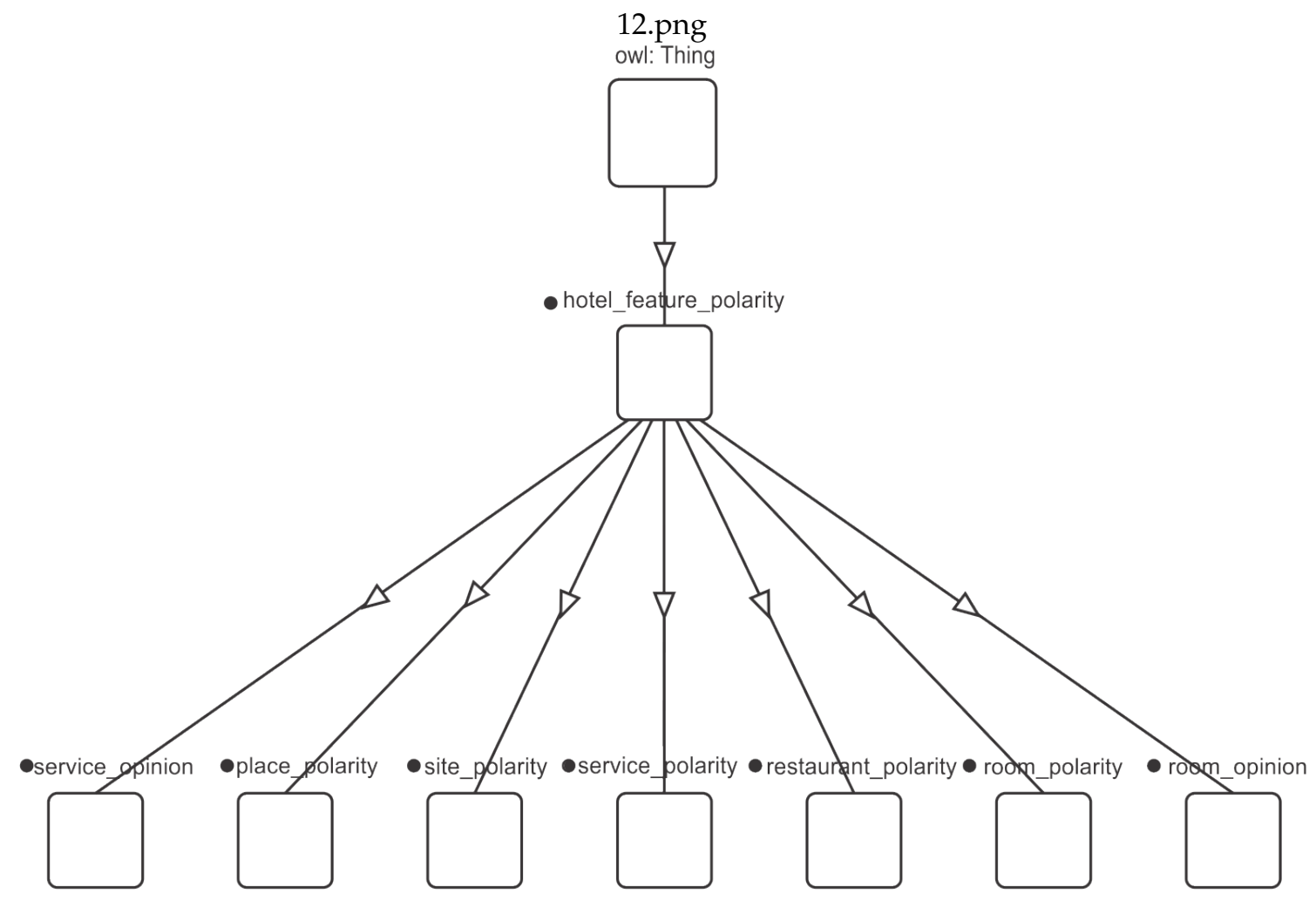

Figure 12. Hotel Class (Ali et al.)

\subsection{Indoor navigation for motion disabled persons in medical facilities}

In the work of Fleiner, Szász, Nagy, and Micsik, 2017 [32] they propose a navigation system to help visitors and patients with disabilities in movements to move in a hospital in Hungary, because there are many complexities within a hospital, since the current navigation systems are based on a map and coordinates by GPS (Global Positioning System). This system posed by the authors is quite economical and uses Linked Open Data (LOD) and SPARQL (Protocol and RDF Query Language) to create flexible APIs (Application Programming Interface) that provide location and routing information.

The complexity of the system requires a data model developed as a formal ontology, which also helps to apply the principles of data linked to published data. In their work the authors provide a data model in the form of two ontologies called: iLOC and hLOC.

- Ontology iLOC. This ontology provides a description of the interior location of a general building, a method of navigation within a building and accessibility attributes for people with disabilities. The iLOC ontology was designed in such a way that it can be easily expanded by additional ontologies to address specific use cases of the environment. The ontology is shown in the figure 13. 


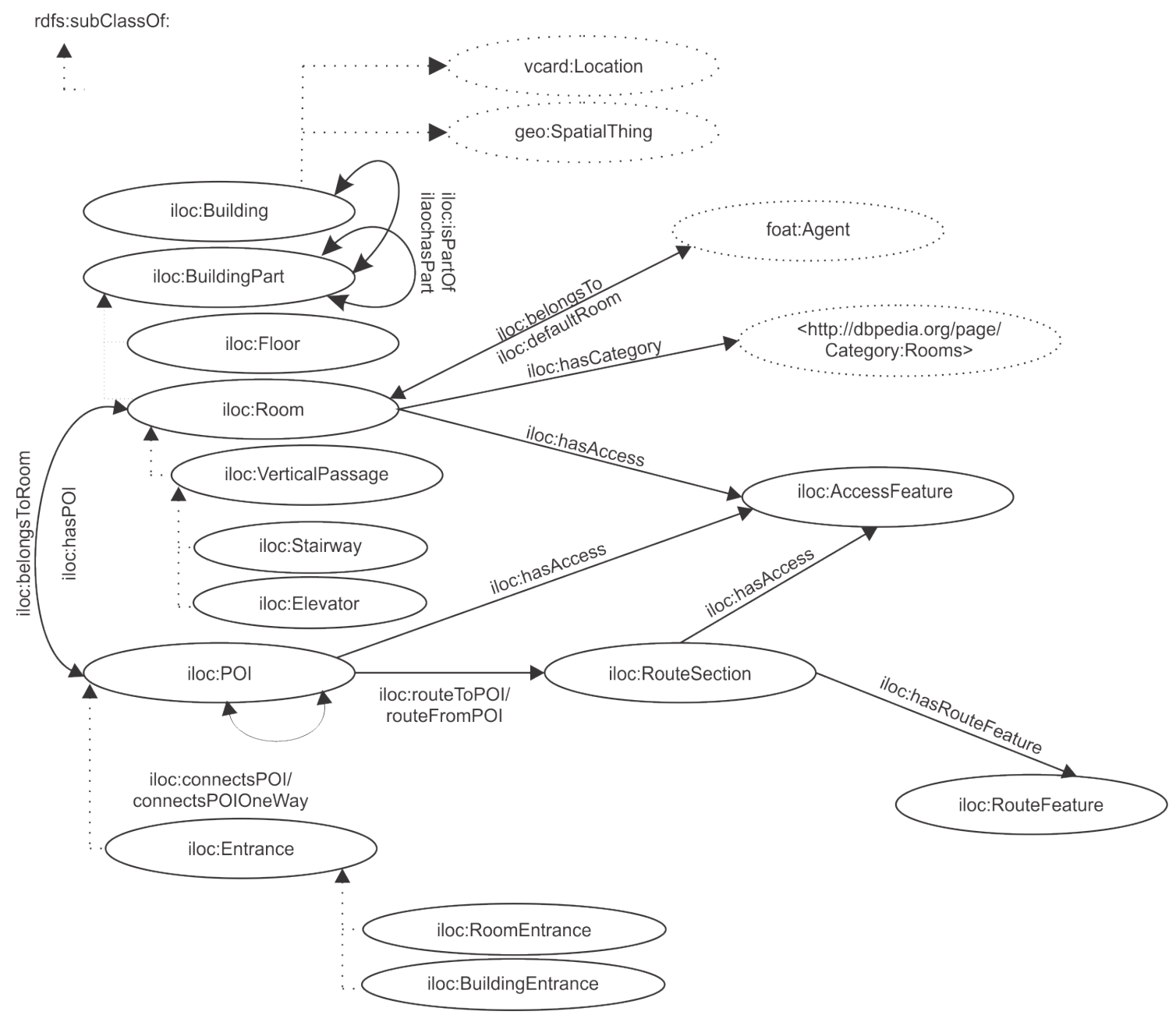

Figure 13. iLOC Ontology (Fleiner, Szász, Nagy, and Micsik)

It is observed that in the iLOC ontology, the AccessFeature class represents different disabilities, which require special features to pass through a section of the hospital. The authors emphasize supporting people with movement disabilities, that is, people with wheelchairs. In the future the authors mention that they can add additional instances to expand other accessibility features.

- hLOC Ontology. The authors mention that the iLOC ontology lacks more features, however, it can be expanded to provide support for indoor environments in hospitals, including support services found in medical facilities. Then hLOC is an extension on iLOC that allows its use in hospitals and provides navigation support for people with movement disabilities.

\subsection{Ontology-Based User Profile Modelling to Facilitate Inclusion of Visual Impairment People}

In the work of Torres, Rodriguez, Espin and Visitacion, 2017 [33] they mention that new technologies are increasingly present in our daily lives because they have characteristics that allow us to access information, entertain, communicate or help in daily life to Users with functional difficulties. In addition, mobile devices include some applications (apps) that facilitate and support different types of tasks or interactions with other users. In many cases, the information provided by these applications is displayed incorrectly because it is not structured, it is presented only graphically and visually without a description of alternative text or descriptive audio, so people who have a visual impairment cannot have access to your content. According to the authors, to provide accessibility of software and hardware, it is necessary to take into account the profile of the users, the physical context and the technology used. From this, the authors focus on people with visual disabilities and their problems when using mobile devices and their applications installed on them. 
To consider the best way to obtain the correct application when performing a search, they have designed a system called m-RECACC [35], which is based on ontologies. This application recommends the best applications according to the user's needs taking into account some features, such as user context, mobile device and accessibility functions.

The authors also mention that it is necessary, due to the global globalization process to have a clear idea about the definition and classification of disabilities using a common language. Complementing the current classification proposed by the World Health Organization (WHO) and the International Classification of Functioning, Disability and Health (ICF), the authors present a new classification proposal that unifies all terms in 4 groups such as: Physical disability, cognitive disability, communication disability and sensory disability. This classification is taken into account to model the profile of users with disabilities.

Based on previous work such as AEGIS, RGD-DO and GUMO, the authors conclude that none of the above ontologies offer a complete user profile according to their proposal such as: personal details of the user, disabilities (classification, grade and diseases) and method and results from the evaluation of the user's disabilities. The option of merging the ontologies of the mentioned works is more difficult. For these reasons the authors create a new ontology according to their requirements. To model all the information related to mobile applications for users with some special needs, 6 ontologies were created that relate: user profile, apps characteristics, context with data extracted from the ambient environment, mobile devices which collects information, accessibility Features provided by mobile devices and languages.

Finally, the merger process was carried out to create a unique ontology to form the knowledge base of the m-RECACC system. The Protégé [36] editor was used as a tool used to build all the mentioned ontologies. The following part of the ontology is presented in the figure 14 .

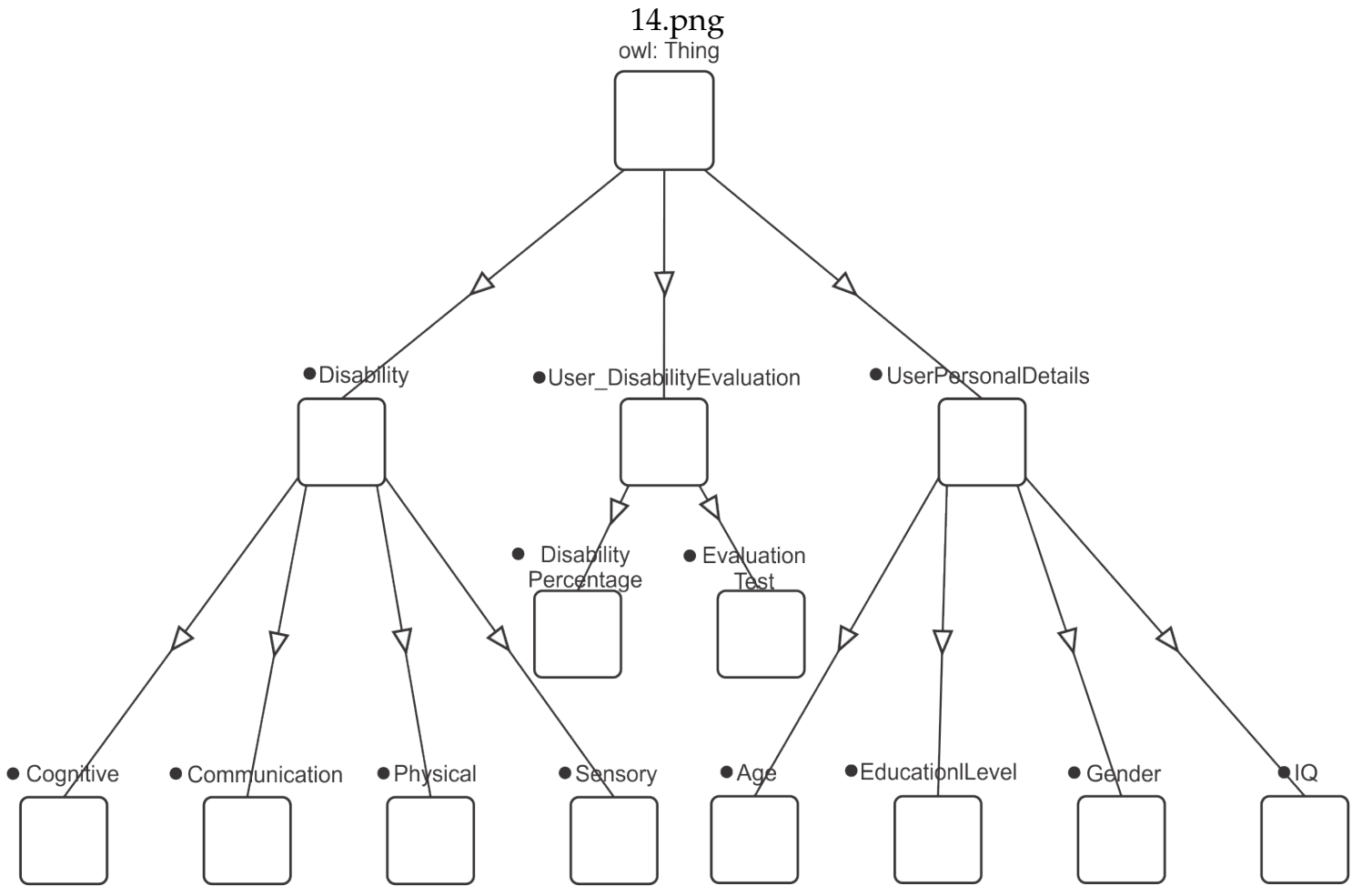

Figure 14. Ontology of User profile (Torres, Rodriguez, Espin and Visitacion)

\subsection{Restful Web Services Composition Using Semantic Ontology for Elderly Living Assistance Services}

In the work of Fattah, and Chong, 2018 [34] they mention that, with the development in medical science, people live longer than previous generations and it is expected that around $20 \%$ of the world population will be 60 years or older for 2050. This will cause many effects, such as the increase in the 
cost of medical care, the increase in illnesses, the shortage of caregivers, etc. The researchers then focus on assistance services for the elderly and disabled in intelligent environments. These environments have intelligent devices interconnected within the network environment and are based on the Internet of Things (IoT). The IoT combines a large number of technologies and comprises a variety of everyday objects interconnected through the Internet and that can interact with each other, where most of its applications are based on services provided by sensors and actuators. Then, an efficient and scalable approach is required to compose services and create models of collaboration between these physical objects.

Taking into account the need for assistance services for older people in smart homes, several solutions have been proposed for the creation of such services. However, each person has different needs. Although technologies are already integrated into everyday life, interoperability between health systems does not achieve an adequate solution. Based on this, the authors propose an intelligent home network architecture based on Web of Objects (WoO) and propose a semantic modeling to support the assistance services for older people to live.

In WoO, real-world entities are represented by virtual objects (VO) and a group of VOs that participate in the performance of a special task is represented by composite virtual objects (CVO). A CVO functions as a service entity in WoO, which allows the creation of support services for the elderly in smart home settings based on the individual requirements of each user. The following figure shows the semantic modeling for the representation of $\mathrm{VO}$ and $\mathrm{CVO}$, for the implementation of the ontology the Protegé development tool was used, the rules in SWRL and SPARQL language are also defined. The following part of the ontology is presented in the figure 15.

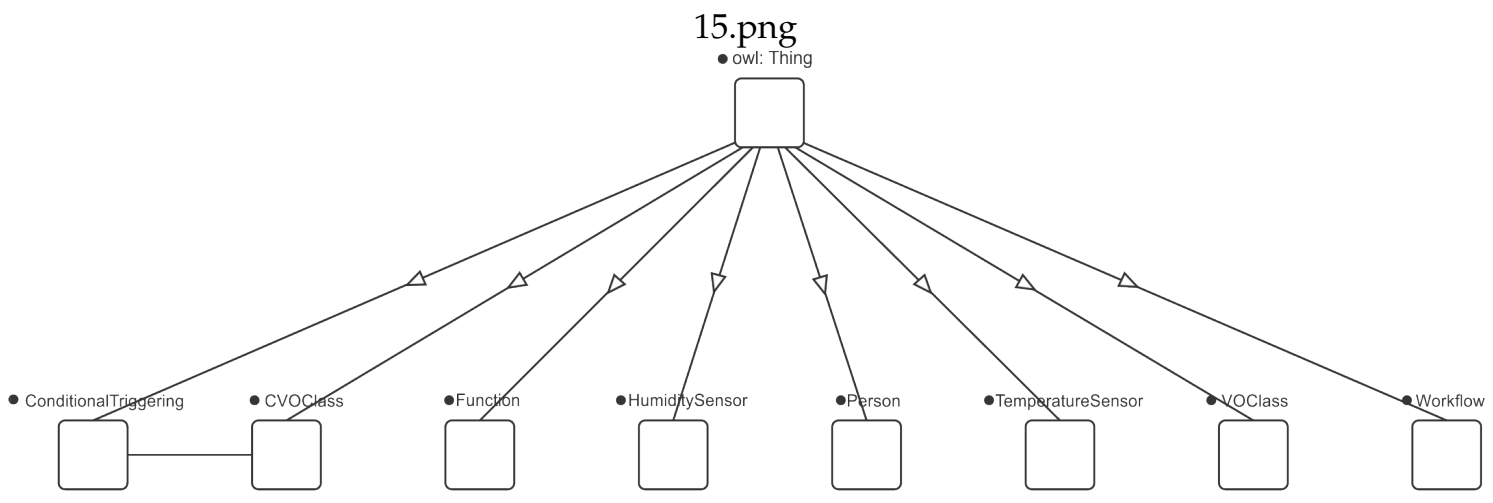

Figure 15. Ontology of RWO (Fattah, and Chong)

\section{Evaluation of case studies}

As our ontology study is based on different domains, but all of them related to people with disabilities, we believe that the ontological models analyzed can be related in some way, indicating the type of person that can help, after their implementation in a system. We can summarize everything in the table 1 . 
Table 1. Ontological frames designed

\begin{tabular}{|c|c|c|}
\hline Work & Ontological frame designed & $\begin{array}{l}\text { To the type of person that could } \\
\text { specifically help }\end{array}$ \\
\hline Kehagias & $\begin{array}{l}\text { Integration of various services: transport, } \\
\text { tourism, and vacations, accesibility to } \\
\text { services, e-learning, e-working and social } \\
\text { relations. }\end{array}$ & Person with physical disability \\
\hline Zakraoui & Web accessibility & For everyone and older adults \\
\hline Nganji & Personalized online teaching (e-learning) & Person with mental and physical disability \\
\hline Gupta & Learning style & Autistic people \\
\hline Bukhari & Information extraction through the web & Person with visual disability \\
\hline Alonso & $\begin{array}{l}\text { Classification and retrieval of information } \\
\text { for tourism on the web }\end{array}$ & For everyone \\
\hline Vassilev & From people assistance on the web & For all and older adults \\
\hline Rivas & $\begin{array}{l}\text { Personalized recommendation for } \\
\text { employment }\end{array}$ & Person with physical disability \\
\hline Zerkouk & $\begin{array}{l}\text { Personalized access to services based on the } \\
\text { user's capabilities and behavior }\end{array}$ & $\begin{array}{l}\text { People with physical disability and older } \\
\text { adults }\end{array}$ \\
\hline Rosales & Of recommendation of tourist sites & Person with visual disability \\
\hline Djaid & Integration in intelligent environments & Person with physical disability \\
\hline Ali & $\begin{array}{l}\text { Integration of various services: transport, } \\
\text { city, hotel and medicines }\end{array}$ & Person with physical disability \\
\hline Fleiner & Navigation in a hospital & Person with physical disability \\
\hline Torres & $\begin{array}{l}\text { Recommendation of the best apps based on } \\
\text { the user's profile }\end{array}$ & Person with visual disability \\
\hline Fattah & $\begin{array}{l}\text { Assistance to the elderly in smart home } \\
\text { environments based on Object Web }\end{array}$ & $\begin{array}{l}\text { People with physical disability and older } \\
\text { adults }\end{array}$ \\
\hline
\end{tabular}

From Table 1 we observe that the efforts made by (Kehagias, Nganji, Rivas, Zerkouk, Djaid, Ali, Fleimer and Fattah) are similar in their contribution because they are aimed at providing help to people with physical disabilities. In the same way, the efforts made by (Bukhari, Rosales and Torres) are similar in their contribution because they are aimed at providing help to people with visual disabilities. Also the work carried out by (Zakraoui, Vassilev, Zerkouk and Fattah)) are similar in their contribution because they are oriented to provide help to adults. Finally, there are also few efforts made to help all types of disabilities.

After implementing the cases studied with the technologies of the Semantic Web, they can be summarized according to the type of accessibility and if there is an ontology for its implementation, in such a way that it allows people with disabilities to help when they want to access some activity of Your daily life. All the mentioned criteria can be summarized in the table 2.

Table 2. Type of existing accessibility

\begin{tabular}{ll}
\hline Accessibility Type & Existing Ontology \\
\hline Web Accessibility & Yes \\
Transport Accessibility & Yes \\
Accessibility to tourism & Yes \\
Accessibility for assistance & Yes \\
Accessibility to e-learning & Yes \\
Accessibility to e-working & Yes \\
Accessibility to social events & Yes \\
Accessibility to learning styles & Yes \\
Accessibility to dialogue & Yes \\
Accessibility to indoor environments in hospital & Yes \\
Accessibility to apps & Yes \\
\hline
\end{tabular}


Table 2 may allow other people to help implement a new system for the realization of other specific objectives in this area, such as being able to reuse the ontology already designed, if necessary. Taking advantage of a knowledge model already worked, including integrating various ontological models in a kind of global ontology or Meta ontology, which allows covering various areas of knowledge in this domain of work, all due to the complex and expensive it would be to design a new ontology.

A factor that is taken into account in most of the articles analyzed is the development of the taxonomy of the case, for a good construction of the ontology. It is also observed in most of the articles analyzed the use of software for the construction of the ontology, it is essential and the one used was the editor Protegé[36]

\section{Conclusions}

The works described in this paper show how knowledge can be modeled in different domains related to people who have some type of disability, using ontologies. In addition, they teach us how this knowledge model, when implemented on the current Web with the tools of the Semantic Web could contribute in the field of accessibility by allowing such people to perform searches on systems taking into account their special needs.

From this analysis we can conclude that some ontological models can be reused for various types of disability depending on the case study. For example, if an online teaching model is desired, one of the existing ones will be searched for their respective analysis and possible implementation. In addition, we observe that some jobs have a broader coverage than others, because they have been developed in larger projects and because they cover more types of disabilities. For example, we observe some ontological models that show its application for all types of people and not only for a special type of disability.

There are even some cases that are disjoint with others. For example, the ontology of learning styles is directed only for autistic people and not for people with visual problems. From comparative table 1 we observe that in the majority of cases there is a heterogeneity in the construction and development of the ontology, however, since it is an issue related to disability, we think that the most important factor would be social acceptance and the proposal of architecture. From this, the ontological model designed would be more valued in our daily lives.

From the analysis of the presented models, some use RDF (Resource Description Framework) and others OWL (Web Ontology Language) as standards for their Semantic Web, in order to provide a framework for the management, integration, sharing and reuse of data on the Web. However, it is observed that the studied models generally use OWL because it provides more vocabulary than RDF Schema to describe its cardinality, properties and classes, as well as the characteristics of its properties, for example the "existing symmetry" between properties. of each model.

Finally, we believe that the usefulness of this study could be that, with the information presented, a Meta ontology could be constructed that includes some or all of the tasks to be used. Then, based on the ontological model, a new system can be built in different areas that covers more activities to be carried out, for example, if a person with a physical disability wishes to do tourism, the designed application should contain the search tasks of tourism sites and also the attention to this person, since this person may find that some tourist sites do not have ramps and cannot access. The designed system should help to choose which sites can be visited, from this a recommendation could be generated to the State so that said tourist site is accessible to all.

Author Contributions: “Conceptualization, J.R. and J.C.; methodology, J.R and J.C.; validation, F.P.; investigation, J.R.; writing-original draft preparation, F.P.; writing-review and editing, A.L.; supervision, L.Z.; project administration, L.Z and A.L. All authors have read and agreed to the published version of the manuscript."

Funding: "This research received no external funding"

Conflicts of Interest: "The authors declare no conflict of interests".

\section{Abbreviations}


The following abbreviations are used in this manuscript:

MDPI Multidisciplinary Digital Publishing Institute

DOAJ Directory of open access journals

TLA Three letter acronym

LD linear dichroism

\section{References}

1. Berners-Lee, T.; Hendler, J.; Lassila, O. The semantic web. Scientific american 2001, 284, 34-43.

2. WHO. https://www.who.int/news-room/fact-sheets/detail/disability-and-health, accessed Feb 3, 2020.

3. AEGIS Project. http://www.aegis-project.eu/, accessed Feb 3, 2020.

4. Shimoyama, M.; De Pons, J.; Hayman, G.T.; Laulederkind, S.J.; Liu, W.; Nigam, R.; Petri, V.; Smith, J.R.; Tutaj, M.; Wang, S.J.; others. The Rat Genome Database 2015: genomic, phenotypic and environmental variations and disease. Nucleic acids research 2015, 43, D743-D750.

5. Basit, K.A.; Matskin, M. GUMO inspired ontology to support user experience based Citywide Mobile Learning. 2011 International Conference on User Science and Engineering (i-USEr). IEEE, 2011, pp. 195-200.

6. Zinnikus, I.; Bogdanovich, A.; Schäfer, R. An ontology based recommendation system for elderly and disabled persons. Workshop Adaptability and User Modeling in Interactive Systems (ABIS 2002), 2002.

7. Bagüés, M.I.; Bermúdez, J.; Illarramendi, A.; Tablado, A.; Goñi, A. Using ontologies in the development of an innovating system for elderly people tele-assistance. OTM Confederated International Conferences" On the Move to Meaningful Internet Systems". Springer, 2003, pp. 889-905.

8. Yesilada, Y.; Harper, S.; Goble, C.; Stevens, R. Screen readers cannot see. International Conference on Web Engineering. Springer, 2004, pp. 445-458.

9. Schwinger, W.; Retschitzegger, W.; Pühretmair, F.; Nussbaum, G. Towards an Ontology-Based Customization Approach for Supporting People with Special Needs. iiWAS. Citeseer, 2005, pp. 185-196.

10. Karim, S.; Tjoa, A.M. Towards the use of ontologies for improving user interaction for people with special needs. International Conference on Computers for Handicapped Persons. Springer, 2006, pp. 77-84.

11. Rudner, M.; Rönnberg, J. Towards a functional ontology for working memory for sign and speech. Cognitive Processing 2006, 7, 183-186.

12. Alfano, B.; Brunetti, A.; De Pietro, G.; Esposito, A. An ontology approach for classification of abnormal white matter in patients with multiple sclerosis. Symposium of the Austrian HCI and Usability Engineering Group. Springer, 2007, pp. 389-402.

13. Latfi, F.; Lefebvre, B.; Descheneaux, C. Ontology-Based Management of the Telehealth Smart Home, Dedicated to Elderly in Loss of Cognitive Autonomy. OWLED, 2007, Vol. 258.

14. Felice, M.; Di Mascio, T.; Gennari, R. A visual ontology-driven interface for a web sign language dictionary. International Conference on Web Information Systems Engineering. Springer, 2007, pp. 429-440.

15. Gennari, R.; Di Mascio, T. An Ontology for a Web Dictionary of Italian Sign Language. WEBIST (2), 2007, pp. 206-213.

16. Pfalzgraf, A.; Pfleger, N.; Schehl, J.; Steigner, J. ODP-Ontology-based Dialogue Platform. Technical report, Technical Report, 2008, SemVox GmbH. http:/ /www. semvox. de/whitepapers ..., 2008.

17. Keet, C.M.; Alberts, R.; Gerber, A.; Chimamiwa, G. Enhancing Web Portals with Ontology-Based Data Access: The Case Study of South Africa's Accessibility Portal for People with Disabilities. OWLED, 2008, Vol. 432.

18. Tang, Y.; De Baer, P.; Zhao, G.; Meersman, R.; Pudkey, K. Towards a Pattern-Driven Topical Ontology Modeling Methodology in Elderly Care Homes. OTM Confederated International Conferences" On the Move to Meaningful Internet Systems". Springer, 2009, pp. 514-523.

19. Lim, E.H.; Liu, J.N.; Lee, R.S. Collaborative content and user-based web ontology learning system. In Knowledge Seeker-Ontology Modelling for Information Search and Management; Springer, 2011; pp. 181-194.

20. Kehagias, D.D.; Tzovaras, D. An ontology-based framework for web service integration and delivery to mobility impaired users. World Summit on Knowledge Society. Springer, 2010, pp. 555-563.

21. Zakraoui, J.; Zagler, W. An ontology for representing context in user interaction for enhancing web accessibility for all 2010. 
22. Nganji, J.T.; Brayshaw, M.; Tompsett, B. Ontology-based e-learning personalisation for disabled students in higher education. Innovation in Teaching and Learning in Information and Computer Sciences 2011, 10, 1-11.

23. Gupta, S.; Garg, D. Ontology Based Information Retrieval for Learning Styles of Autistic People. International Conference on High Performance Architecture and Grid Computing. Springer, 2011, pp. 293-298.

24. Bukhari, A.C.; Kim, Y.G. Ontology-assisted automatic precise information extractor for visually impaired inhabitants. Artificial Intelligence Review 2012, 38, 9-24.

25. Alonso, K.; Aginako, N.; Lozano, J.; Olaizola, I.G. Ontology based middleware for ranking and retrieving information on locations adapted for people with special needs. International Conference on Computers for Handicapped Persons. Springer, 2012, pp. 351-354.

26. Vassilev, V.; Ulman, M.; Ouazzane, K.; Kazemian, H.; Aigbodi, M.; Boyd, R. Ontocarer: An ontological framework for assistive agents for the disabled. The 3rd International Conference on Digital Information Processing and Communications (ICDIPC2013), 2013, pp. 404-416.

27. Rivas-Costa, C.; Anido-Rifón, L.; Fernández-Iglesias, M.J.; Gómez-Carballa, M.A.; Valladares-Rodríguez, S.; Soto-Barreiros, R. An accessible platform for people with disabilities. International Journal of Human-Computer Interaction 2014, 30, 480-494.

28. Zerkouk, M.; Cavalcante, P.; Mhamed, A.; Boudy, J.; Messabih, B. Behavior and capability based access control model for personalized telehealthcare assistance. Mobile Networks and Applications 2014, 19, $392-403$.

29. Rosales-Huamaní, J.; Castillo-Sequera, J.; Puente-Mansilla, F.; Boza-Quispe, G.; others. A Prototype of a Semantic Platform with a Speech Recognition System for Visual Impaired People. Journal of Intelligent Learning Systems and Applications 2015, 7, 87.

30. Djaid, N.T.; Dourlens, S.; Saadia, N.; Ramdane-Cherif, A. Fusion and fission engine for an assistant robot using an ontology knowledge base. Journal of Ambient Intelligence and Smart Environments 2017, 9, 757-781.

31. Ali, F.; Kwak, D.; Khan, P.; Ei-Sappagh, S.H.A.; Islam, S.R.; Park, D.; Kwak, K.S. Merged ontology and SVM-based information extraction and recommendation system for social robots. IEEE Access 2017, 5, 12364-12379.

32. Fleiner, R.; Szász, B.; Simon-Nagy, G.; Micsik, A. Indoor navigation for motion disabled persons in medical facilities. Acta Polytechnica Hungarica 2017, 14, 111-128.

33. Torres-Carazo, M.I.; Rodríguez-Fórtiz, M.J.; Espin-Martin, V.; Hurtado, M.V. Ontology-Based User Profile Modelling to Facilitate Inclusion of Visual Impairment People. International Conference on Model and Data Engineering. Springer, 2017, pp. 386-394.

34. Fattah, S.M.M.; Chong, I. Restful Web Services Composition Using Semantic Ontology for Elderly Living Assistance Services. Journal of Information Processing Systems 2018, 14.

35. Torres-Carazo, M.I.; Rodríguez-Fórtiz, M.J.; Hurtado, M.V.; Samos, J.; Espín, V. Architecture of a mobile app recommender system for people with special needs. International Conference on Ubiquitous Computing and Ambient Intelligence. Springer, 2014, pp. 288-291.

36. Protégé. https:// protege.stanford.edu, accessed Feb 3, 2020.

Sample Availability: Samples of the compounds ...... are available from the authors. 